\title{
Global analysis of parallel analog networks with retarded feedback
}

\author{
A. V. M. Herz \\ Division of Chemistry, California Institute of Technology 139-74, Pasadena, California 91125
}

(Received 6 March 1991)

\begin{abstract}
We analyze the retrieval dynamics of analog "neural" networks with clocked sigmoid elements and multiple signal delays. Proving a conjecture by Marcus and Westervelt, we show that for delayindependent symmetric coupling strengths, the only attractors are fixed points and periodic limit cycles. The same result applies to a larger class of asymmetric networks that may be utilized to store temporal associations with a cyclic structure. We discuss implications for various learning schemes in the spacetime domain.
\end{abstract}

Signal transmission processes play an important role for the dynamics of the nervous system [1]. The underlying electrochemical transport mechanisms are quite intricate and cannot be modeled explicitly within the mesoscopic approach [2] to biological information processing. On this level of description, single neutrons are treated as elementary building blocks, most commonly in the form of binary threshold units or sigmoid analog elements, and signal propagation processes are incorporated into the dynamical description as retarded interactions [3]. $\mathrm{Nu}$ merical and analytical studies show that a chaotic time evolution is rather common in systems with delayed feedback - in single cells [4] as well as in large assemblies [5].

In contrast to the above observation, some neurobiological central pattern generators utilize delayed feedback to initiate precise rhythmic activity of motor neutrons [6]. Furthermore, artificial networks with retarded interactions have proven to operate very effectively as associative memories in the space-time domain once special configurations of synaptic coupling strengths have been implemented by hand $[7,8]$ or memorized by some appropriate learning rule [9-12]. However, even these systems exhibit irregular temporal behavior for certain parameter values $[13,14]$, greatly affecting their capabilities as content addressable memories.

It is therefore of broad interest to determine general conditions which exclude spurious chaotic motion in delayed-feedback networks but still allow for nontrivial spatiotemporal phenomena. As is well known, a local stability analysis cannot deal with this question [15], and instead, global techniques have to be applied.

So far, two similar approaches have been reported: For systems with binary elements and a parallel deterministic updating rule, a Lyapunov functional for the retrieval dynamics has been introduced in Ref. [16]. It generalizes Hopfield's concept [17] of an "energy landscape" to the space-time domain, applies to a wide class of networks that store both static patterns and temporal associations, and assures that the dynamics relaxes to fixed points or periodic limit cycles. A slightly different route has been taken by Marcus and Westervelt [18]. These authors have focused on systems with analog elements and delay-independent symmetric interactions. Extending prior work on graded-response neurons $[19,20]$ and iterated maps [21], they showed that clocked networks converge to fixed-point attractors under certain conditions for the synaptic strengths. Guided by some evidence from systems with small maximum delay, they conjectured that if those restrictions for the synaptic weights are not met, the only new attractors are periodic limit cycles.

In the present paper, essential ingredients of both approaches are combined to form a more general framework for the study of analog networks. We focus on synchronous systems, introduce a novel Lyapunov functional, and prove the conjecture of Marcus and Westervelt. We then turn to a larger group of asymmetric networks and discuss both unsupervised and teacher-forced learning schemes for temporal sequences.

The time evolution of analog networks with a synchronous updating rule is defined by a set of coupled difference equations,

$$
V_{i}(t+1)=g_{i}\left(h_{i}(t)\right)
$$

with

$$
h_{i}(t)=\sum_{j=1}^{N} \sum_{\tau=0}^{\tau_{\max }} J_{i j}(\tau) V_{j}(t-\tau)+I_{i}(t) .
$$

Here, $V_{i}$ denotes the activity of the $i$ th cell, $1 \leq i \leq N$, and depends through $g_{i}$ on the local field (postsynaptic potential) $h_{i}$, which in turn is determined by the external input $I_{i}$ and previous neural activities, weighted by the synaptic coupling strengths $J_{i j}(\tau)$. For simplicity, the basic time step (clock cycle) has been set equal to unity. Consequently, the signal delays take nonnegative integer values. For the special case of delay-independent symmetric weights, i.e., $J_{i j}(\tau)=J_{i j}=J_{j i}$ for $0 \leq \tau \leq \tau_{\max }$, we recover the description of Ref. [18]

Throughout the present paper, it is generally assumed that the input-output characteristics $g_{i}$ are single valued, monotonically increasing, and differentiable, so that there are well-defined inverse functions $g_{i}^{-1}$ with the same properties. We will call transfer functions $g_{i}$ "sigmoid," if in addition they also grow in magnitude more slowly than linear for large positive or negative arguments. We introduce auxiliary quantities $G_{i}\left(V_{i}\right)$, 


$$
G_{i}\left(V_{i}\right) \equiv \int_{0}^{V_{i}} d x g_{i}^{-1}(x),
$$

which for sigmoid $g_{i}$ and large absolute $V_{i}$ increase faster then $V_{i}^{2}$ by construction. Following the notation of Ref. [18], we denote the maximum slope of $g_{i}$ by $\beta_{i}$.

We now prove that there is a Lyapunov functional for the dynamical system (1),(2) if the synaptic couplings exhibit an "extended synaptic symmetry" [21] for some (fixed) positive integer $D$,

$$
J_{i j}(\tau)=\left\{\begin{array}{l}
J_{j i}(\tau) \text { for } \tau=D-1 \\
J_{j i}(D-\tau-2) \text { for } 0 \leq \tau<D-1 .
\end{array}\right.
$$

Links with $\tau \geq D$ are assumed to vanish, i.e., $\tau_{\max }<D$. Notice that the coupling strengths might be asymmetric for $\tau \neq D-1$ in the sense that $J_{i j}(\tau) \neq J_{j i}(\tau)$.

Consider the functional

$H(t)=-\frac{1}{2} \sum_{i, j=1}^{N} \sum_{a, \tau=0}^{D-1} J_{i j}(\tau) V_{i}(t-a) V_{j}(t-(a+\tau+1) \bmod D)+\sum_{i=1}^{N} \sum_{\tau=0}^{D-1}\left[G_{i}\left(V_{i}(t-\tau)\right)-I_{i}(t-1-\tau) V_{i}(t-\tau)\right]$

External inputs are supposed to be $D$ periodic, a condition that is satisfied in a trivial way for the standard case of timeindependent inputs. The difference $\Delta H(t) \equiv H(t)-H(t-1)$ is then given by

$$
\Delta H(t)=-\sum_{i=1}^{N} \Delta Z_{i}(t) h_{i}(t-1)-\frac{1}{2} \sum_{i, j=1}^{N} J_{i j}(D-1) \Delta Z_{i}(t) \Delta Z_{j}(t)+\sum_{i=1}^{N}\left[G_{i}\left(V_{i}(t)\right)-G_{i}\left(V_{i}(t-D)\right)\right],
$$

where

$$
Z_{i}(t) \equiv \sum_{\tau=0}^{D-1} V_{i}(t-\tau)
$$

and

$$
\Delta Z_{i}(t) \equiv Z_{i}(t)-Z_{i}(t-1)=V_{i}(t)-V_{i}(t-D) .
$$

The right-hand side of $(6)$ is zero if $\Delta Z_{i}(t)=0$ for all $i$.

In what follows, we analyze the case where $V_{i}(t) \neq V_{i}(t-D)$ for at least some $i$. Since $g_{i}^{-1}$ is single valued and monotonically increasing by assumption, $G_{i}$ is strictly convex. Through a Taylor expansion of $G_{i}\left(V_{i}(t-D)\right)$ around $G_{i}\left(V_{i}(t)\right)$, we obtain the following upper bound for those $i$ in the last term of (6):

$$
G_{i}\left(V_{i}(t)\right)-G_{i}\left(V_{i}(t-D)\right)<\Delta Z_{i}(t) G_{i}^{\prime}\left(V_{i}(t)\right)-\frac{1}{2} \Delta Z_{i}(t)^{2} G_{i}^{\prime \prime}\left(V_{i}(t)\right) \leq \Delta Z_{i}(t) h_{i}(t-1)-\frac{1}{2} \Delta Z_{i}(t)^{2} \beta_{i}^{-1} .
$$

For a graphical illustration see Ref. [18]. Inserting (8) into (6), we arrive at

$$
\begin{aligned}
\Delta H(t)< & -\frac{1}{2} \sum_{i, j=1}^{N} J_{i j}(D-1) \Delta Z_{i}(t) \Delta Z_{j}(t) \\
& -\frac{1}{2} \sum_{i=1}^{N} \beta_{i}^{-1} \Delta Z_{i}(t)^{2} .
\end{aligned}
$$

A sufficient condition for $\Delta H(t)<0$ is therefore given by

$$
\lambda_{\min }[\mathbf{J}(D-1)] \geq-\beta^{-1},
$$

where $\lambda_{\min }[\mathbf{J}(D-1)]$ denotes the smallest eigenvalue of the matrix $\mathbf{J}(D-1)$ with components $J_{i j}(D-1)$ and $\beta \equiv \max _{i}\left\{\beta_{i}\right\}$. As long as $\Delta Z_{i}(t) \neq 0$ for some $i, H$ has to decrease if (10) is met. Since $H$ is bounded from below for sigmoid $g_{i}, \Delta H(t)$ and $\Delta Z_{i}(t)$ vanish asymptotically for large $t$.

We have thus demonstrated that the retrieval dynamics of synchronized analog networks with sigmoid elements and retarded feedback is governed by a Lyapunov functional if the synaptic weights satisfy both (4) and (10). Furthermore, we have shown that the network relaxes to a solution with $V_{i}(t)=V_{i}(t-D)$, i.e., to a static state or a limit cycle with a period equal to $D$ or an integer fraction of $D$.
The model of Ref. [16] uses two-state threshold units $V_{i}= \pm 1$ with a parallel stochastic time evolution, $\operatorname{Prob}\left[V_{i}(t+1)= \pm 1\right]=\frac{1}{2}\left\{1 \pm \tanh \left[\beta h_{i}(t)\right]\right\}$. Here, $\beta^{-1}$ denotes the noise level and $h_{i}(t)$ is given by (2) as in the continuous case. The deterministic limit, $V_{i}(t+1)=\operatorname{sgn}\left[h_{i}(t)\right]$, may also be regarded as a highgain limit of the analog network if we consider inputoutput characteristics $g_{i}$ that saturate at $+1(-1)$ for large positive (negative) arguments, e.g., $g_{i}\left(h_{i}\right)$ $=\tanh \left(\beta_{i} h_{i}\right)$. The relationship between the discrete and the continuous system is therefore similar to that between the original Hopfield model [17] with random sequential stochastic updates and its graded-response counterpart [20], operating in continuous time. This resemblance carries over to the Lyapunov functionals as well, where for $\beta_{i} \rightarrow \infty$, the contribution of $G_{i}$ to (5) can be neglected, leaving the functional for the Ising-spin model with distributed delays.

Returning to analog systems, we now discuss some results for two special synaptic configurations. First, we choose delay-independent symmetric couplings and $\tau_{\max }=D-1$,

$$
J_{i j}(\tau)=J_{i j}=J_{j i} \text { for } 0 \leq \tau \leq D-1 .
$$

The temporal sums in (5) decouple and we obtain 


$$
\begin{aligned}
H(t)= & -\frac{1}{2} \sum_{i, j=1}^{N} J_{i j} Z_{i}(t) Z_{j}(t) \\
& +\sum_{i=1}^{N} \sum_{\tau=0}^{D-1}\left[G_{i}\left(V_{i}(t-\tau)\right)\right. \\
& \left.-I_{i}(t-1-\tau) V_{i}(t-\tau)\right] .
\end{aligned}
$$

This expression is equivalent to the first Lyapunov functional of Ref. [18] where it is shown that if (10) and (11) hold, there are fixed-point attractors only. With properly normalized couplings, $J_{i j} \propto D^{-1}$, these solutions are identical to the attractors of the graded-response model [20], however, regions of attraction and convergence times are changed [18].

Second, we consider a slight modification of Eq. (11),

$$
J_{i j}(\tau)=\left\{\begin{array}{l}
0 \text { for } \tau=D-1 \\
J_{i j}=J_{j i} \text { for } 0 \leq \tau<D-1 .
\end{array}\right.
$$

Equation (10) is now satisfied for all sigmoid $g_{i}$. We have thus proven the conjecture of Marcus and Westervelt [18]: For synchronous analog networks with arbitrary sigmoid input-output functions, symmetric couplings and maximum delay $D-2$, all attractors are restricted to be limit cycles with periods of $D$ and its divisors.

In passing $I$ would like to note that if $D$ is replaced by $D+1$ in (13), both (11) and (13) describe the same network. However, the corresponding Lyapunov functionals differ even for constant external inputs, and it is only for the second case that one may draw the above conclusions. Furthermore, it is worthwhile to mention that the second Lyapunov functional of Ref. [18] follows without guesswork as a special case from (5) and (13) for $D=3$.

For the remaining part of the paper, we turn to more general coupling matrices and discuss the storage of temporal associations with common duration $D$. Since we know that the retrieval dynamics has to converge to $D$ periodic limit cycles once the conditions (4) and (10) are met, the main task is to construct or learn synaptic couplings which guarantee that these limit cycles indeed resemble the desired spatiotemporal attractors for appropriate initial conditions. Similar to the static case, various approaches may be pursued. I will focus on one unsupervised learning rule and briefly comment on some teacher-forced scenarios.

Generalizing from networks with binary elements [16], let us take

$$
J_{i j}(\tau)=\epsilon(\tau) \frac{1}{N} \sum_{\mu=1}^{P} \sum_{t_{\mu}=0}^{D-1} V_{i}\left(t_{\mu}+1\right) V_{j}\left(\left(t_{\mu}-\tau\right) \bmod D\right)
$$

where $\mu$ labels the $P$ sequences $\left\{V_{i}\left(t_{\mu}\right) ; 1 \leq i \leq N\right.$, $\left.1 \leq t_{\mu} \leq D\right\}$. The parameters $\epsilon(\tau)$ may be used to define various network architectures [10]. The coupling strengths will, in general, be asymmetric, $J_{i j}(\tau) \neq J_{j i}(\tau)$, but for all networks with $\epsilon(\tau)=\epsilon[D-(2+\tau) \bmod D]$, the "extended synaptic symmetry" (4) still holds. For simplicity, we set $\epsilon(D-1)=0$ to satisfy (10) as well.

As a result of the modulo operation in Eq. (14), arbitrary sequences are memorized as if they were $D$-periodic cycles. They will be retrieved accordingly. If during learning, a sequence is already presented $\tau_{\max }$ time steps before synaptic strengths are altered, the modulo operation may be dropped, leaving a learning rule that is Hebbian [22] in the sense that it correlates presynaptic activity with subsequent postsynaptic response - in our case the response is delayed by $\tau+1$ time steps due to both the signal lag and the additional step of the discrete-time dynamics (1).

For sequences of uncorrelated, binary random patterns $V_{i}\left(t_{\mu}\right)= \pm 1$, quantitative analytical and numerical results about the network performance have been obtained in the high-gain limit $\beta_{i} \rightarrow \infty$ [16]. They show that the storage capacity for temporal associations is comparable to that for static memories in the Hopfield model. Arbitrary sigmoid input-output functions may be treated in a similar fashion if one combines the concept of equivalent static systems [16] with a statistical mechanical analysis of graded-response neurons [23].

The learning rule (14) may be utilized to store cycles of correlated real-valued patterns as well. First numerical studies have been performed for low-dimensional trajectories (small $N$ ) with high numbers of data points (large $D$ ). For many examples, good retrieval could be obtained this way [24] - without any need for highly timeconsuming supervised learning schemes [25] - however, teacher-forced algorithms might eventually be necessary to solve more sophisticated tasks. Here, once again, the existence of a Lyapunov functional is of great help since it allows for an application of mean-field techniques [26] to a wide class of supervised learning strategies like spatiotemporal extensions of the "Boltzmann Machine" concept [27] or, more general, contrastive-learning schemes $[28,29]$.

Concerning hardware applications, let us note that the dynamical system (1),(2) can readily be implemented with charge-coupled devices. There, the vector-matrix multiplication in Eq. (2) can be accomplished in a fully parallel fashion at a clock rate in the megahertz regime [30], allowing for fast systematic experiments of adaptive learning schemes. Our theoretical analysis might help to guide and complement such work.

In summary, we have studied the global dynamics of parallel networks with analog elements and retarded feedback. Using a novel Lyapunov functional it could be proven that for a wide class of system architectures and transfer functions, the only attractors are fixed points and certain periodic limit cycles. The results can be regarded as design criteria for artificial networks as well-they show how to extend well-known learning schemes to the spatiotemporal domain and guarantee the absence of spurious chaotic trajectories.

The present study has been triggered by earlier collaborations with Leo van Hemmen, Reimer Kühn, Zhaoping $\mathrm{Li}$, and Bernhard Sulzer. The author also benefited from various activities within their Computation and Neural Systems program at the California Institute of Technology. It is a great pleasure to thank Pierre Baldi, John Hopfield, Marcus Mitchell, and Chuck Neugebauer for valuable discussions. This work has been supported by the Office of Naval Research Contract No. N0001487-K-0377 and by a grant from the Studienstiftung des Deutschen Volkes. 
[1] See, for example, S. W. Kuffler, J. G. Nicholls, and A. R. Martin, From Neuron to Brain (Sinauer, Sunderland, 1984), p. 99ff.

[2] For an overview see D. J. Amit, Modeling Brain Function: The World of Attractor Neural Networks (Cambridge University Press, Cambridge, 1989); J. Hertz, A. Krogh, and R. G. Palmer, Introduction to the Theory of Neural Computation (Addison-Wesley, Redwood City, 1991); Physics of Neural Networks, edited by E. Domany, J. L. van Hemmen, and K. Schulten (Springer, Heidelberg, 1991).

[3] E. Caianiello, J. Theor. Biol. 1, 204 (1961).

[4] K. Aikara, T. Takabe, and M. Toyoda, Phys. Lett. A 144, 333 (1990).

[5] M. Y. Choi and B. A. Huberman, Phys. Rev. B 31, 2862 (1985).

[6] P. A. Getting, J. Neurophysiol. 49, 1036 (1983).

[7] D. Kleinfeld, Proc. Natl. Acad. Sci. U.S.A. 83, 9469 (1986).

[8] H. Sompolinsky and I. Kanter, Phys. Rev. Lett. 57, 2861 (1986).

[9] A. C. C. Coolen and C. C. A. M. Gielen, Europhys. Lett. 7, 281 (1988).

[10] A. V. M. Herz, B. Sulzer, R. Kühn, and J. L. van Hemmen, Europhys. Lett. 7, 663 (1988); Biol. Cybern. 60, 457 (1989).

[11] M. Kerzberg and A. Zippelius, Phys. Scr. T 33, 54 (1990).

[12] K. Bauer and U. Krey, Z. Phys. B Condens. Matter 79, 461 (1990)

[13] U. Riedel, R. Kühn, and J. L. van Hemmen, Phys. Rev. A 38, 1105 (1988).

[14] T. Fukai and M. Shiino, Phys. Rev. Lett. 64, 1465 (1990).

[15] C. M. Marcus and R. M. Westervelt, Phys. Rev. A 39, 347 (1989).
[16] Z. Li and A. V. M. Herz, in Proceedings of the XI Sitges Conference on Neural Networks, edited by L. Garrido, Lecture Notes in Physics Vol. 368 (Springer-Verlag, Berlin, 1990), pp. 287-302; A. V. M. Herz, Z. Li, and J. L. Van Hemmen, Phys. Rev. Lett. 66, 1370 (1991).

[17] J. J. Hopfield, Proc. Natl. Acad. Sci. U.S.A. 79, 2554 (1982).

[18] C. M. Marcus and R. M. Westervelt, Phys. Rev. A 42 , 2410 (1990)

[19] M. A. Cohen and S. Grossberg, IEEE Trans. SMC 13, 815 (1983).

[20] J. J. Hopfield, Proc. Natl. Acad. Sci. U.S.A. 81, 3088 (1984).

[21] C. M. Marcus and R. M Westervelt, Phys. Rev. A 40, 501 (1989).

[22] D. O. Hebb, The Organization of Behavior (Wiley, New York, 1949), p. 62.

[23] R. Kühn, in Proceedings of the XI Sitges Conference on Neural Networks (Ref. 15), pp. 19-32; R. Kühn, S. Bös, and J. L. van Hemmen, Phys. Rev. A 43, 2084 (1991).

[24] A. V. M. Herz and M. Q. Mitchell (unpublished).

[25] B. A. Pearlmutter, Neural Comput. 1, 263 (1989).

[26] C. Peterson and J. R. Anderson, Complex Systems 1, 995 (1987).

[27] D. H. Ackley, G. E. Hinton and T. J. Sejnowski, Cognitive Sci. 9, 147 (1985).

[28] J. Movellan, Proceedings of the 1990 Carnegie Mellon Summer School (Morgan Kaufmann, San Mateo, 1991).

[29] F. Pineda and P. Baldi (unpublished).

[30] A. Agranat, C. F. Neugebauer, and A. Yariv, Appl. Opt. 27, 4354 (1988); C. F. Neugebauer and A. Yariv (unpublished). 